\title{
Snoring: The Case for Molecular Tissue Oximetry
}

\author{
Guy M. Hatch, $\mathrm{MD}^{1}$, Liza Ashbrook, $\mathrm{MD}^{2}$, Aric A. Prather, Ph.D², Andrew D. Krystal, MD, MS ${ }^{2}$ \\ ${ }^{1}$ Reveal Biosensors, Inc, Logan, Utah, USA \\ ${ }^{2}$ University of California San Francisco, San Francisco, CA, USA
}

Guy.Hatch@revealbiosensors.com

\begin{abstract}
Snoring gets no respect. It also gets little to no medical therapy. Why is this? How can something that is clearly pathological based on epidemiologic research not be diagnosed and treated with effective therapy? The problem is the lack of a credible, objective index of pathology during snoring. Pulse oximetry detects drops in arterial blood oxygen saturation $\left(\mathrm{SpO}_{2}\right)$ associated with obstructive sleep apnea and hypopnea events in polysomnographic (PSG) testing. When no desaturation is present, evidence of sleep disturbance is required to indicate the presence of pathology. However, obstruction at the mild end of the continuum of sleep disordered breathing (SDB) can occur without producing a drop in $\mathrm{SpO}_{2}$ or sleep disturbance; in which case it is referred to as 'primary snoring.' Although statistically associated with comorbidities of $\mathrm{SDB}$, without there being a drop in $\mathrm{SpO}_{2}$ or sleep disturbance, primary snoring is not thought to be pathologic enough to warrant diagnosis or treatment. One promising means of detecting the pathological processes associated with primary snoring is molecular tissue oximetry, which detects skin tissue oxygen need, vs. oxygen supply. In cases of hypoxic challenge, regulatory mechanisms restrict blood flow to the skin to preferentially maintain blood oxygen supply to more vital organs. As a result, molecular tissue oximetry of the skin is a more sensitive measure of inadequate breathing than pulse oximetry measuring blood oxygen saturation. In this article we review molecular tissue oximetry technology and methodology and make the case that it is a promising tool for identifying pathology occurring in association with primary snoring.
\end{abstract}

Keywords—Tissue hypoxia; sleep disordered breathing; primary snoring; molecular tissue oximeter; tissue hyperoxia

\section{INTRODUCTION}

Snoring is the subject of countless jokes, emphatic complaints from children and spouses, and is a major, but largely ignored medical problem. The presence of pathology in sleep disordered breathing (SDB) is currently identified during polysomnography (PSG) by the presence of a drop in arterial oxygen saturation, as measured with pulse oximetry, or electroencephalographic (EEG) evidence of sleep disturbance $[1,2,3,4,5]$. These findings are used as the basis for the widely employed definitions for apneas, hypopneas, and respiratory related arousals, which form the basis of the diagnosis of SDB and determine whether to institute therapy $[1,3,4,5]$. By definition, 'primary snoring' is snoring without blood oxygen desaturation and EEG-based arousals [1]. Without measurable indicators of pathology, there is no basis for instituting medical treatment.

However, there is clear evidence that primary snoring is associated with significant medical morbidity, indicating that it is indeed pathological. Primary snoring during sleep is currently thought to exist at the mild, non-pathological, end of a continuum of airway obstruction with medically-recognized obstructive sleep apnea (OSA) at the extreme end. Primary snoring has long been statistically linked to significantly increased risk of hypertension [6], heart disease and stroke [7], premature births [8], and in children, to developmental delay, attention deficit disorder, and socialization and learning disabilities [9]. Recently reported research has also identified two blood pressure-related phenomena linked to snoring; (1) lack of normal blood pressure 'dipping,' [11] and (2) the occurrence of a blood pressure surge following snoring episodes [12]. There are also data suggesting that hypertension may become resistant, or even refractory, to pharmacologic therapy due to chronic sympathetic stimulation from snoring [13]. There is also evidence for subtle sleep disturbance from the partial obstruction of the upper airway that increases the work of breathing. If the level of work needed to breathe through a restricted airway is greater than can be performed while asleep, the person's sleep is either disturbed, or the person may become tolerant of the stress because of increasing sleep deprivation. There is also a possibility that such a mild hypoxic challenge would trigger reduced perfusion of the skin, thereby conserving oxygen consumption for more vital organs, which may prevent or minimize a decrease in blood oxygen saturation [14]. Although there is no evidence that EEG arousals occur with primary snoring, recent data indicate that there is an elevated arousal threshold in snorers that indirectly shows that some sleep disturbance does occur with primary snoring and leads to a degree of sleep deprivation [10].

Despite the epidemiologic evidence for a link to the negative medical outcomes described above, without some objective evidence of pathology proximal to the snoring itself, there is currently no basis for instituting therapy for primary snoring that might prevent the occurrence of these statistically-associated long-term morbidities. Detecting snoring-associated pathology would require a biometric that is (1) scientifically valid, (2) more sensitive to hypoxic 
stress than pulse oximetry, and/or (3) more sensitive to sleep disturbance than EEG. Such a measure would allow documentation of pathology, and the possibility of mitigating the consequences of primary snoring through effective therapy. A new biometric that presents such a promise is molecular tissue oximetry.

\section{PULSE OXIMETRY VS. MOLECULAR TISSUE OXIMETRY}

Pulse oximetry, developed in 1972, is the product of the search to find a continuous, non-invasive alternative to periodic sampling and laboratory testing of arterial blood to track the oxygen content of blood during surgical anesthesia and intensive care. This blood oxygen information is clinically useful when dealing with several major diseases and abnormal cardiovascular conditions. The ability of pulse oximetry to continuously and painlessly monitor this vital physiologic information quickly gained favor with intensive care clinicians who were initially reluctant to accept the relatively less precise new method. The key to the successful adoption came with the discovery of new insights about the dynamic nature of blood oxygen status that were not evident with timed, and even somewhat staged, blood-gas sample timing during surgical anesthesia and intensive care. Pulse oximetry has since become an indispensable tool in numerous areas of medical practice.

Pulse oximeters measure the light intensity at the peak and trough of photoplethysmogram (PPG) waveforms at red and infrared wavelengths of light after the light has passed through tissue, such as a fingertip. The less than $2 \%$ of full scale (FS) pulsatile waveforms (AC) are the portions of the light signals that are most affected by the oxygen saturation of hemoglobin in the arterioles within the light path. Ratio, logarithmic, and Fast Fourier Transform (FFT) calculations are currently used to produce $\mathrm{SpO}_{2}$ percentage output data from these $\mathrm{AC}$ measurements. However, these calculation methods either do not include, or mathematically dispense with, the remaining about $98 \%$ FS of the red and infrared light signals that are non-pulsating (DC).

In contrast, the Reveal molecular tissue oximeter signals are similar to the previously unused $98 \%$, non-pulsating DC components of pulse oximeter signals. At the time of their discovery, the DC signal responses to breathing nitrogen did not appear to have an immediately useful application. However, with the development of a USB cable-attached sensor prototype that could be mounted on the upper arm, it was possible to study skin tissue hypoxia resulting from a variety of stresses. Using this device, we also discovered additional, unique photonic responses to skin tissue hyperoxia induced by breathing oxygen, and complex patterns of response during and following physical exercise. Data from this series of experiments indicates that the molecular tissue oximeter sensor is detecting skin tissue redox status that, under certain conditions, differs from the $\mathrm{SpO}_{2}$ indication of arterial blood oxygen saturation.

This dissociation between pulse oximetry and molecular tissue oximetry has been found to arise in multiple settings, likely some associated with pathology and others without. The difference appears to be a function of context. Normal adaptive stress responses, such as reduction in skin perfusion during moderate exertion, are not pathologic and may be an integral part of the health benefits of exercise. However, if this occurs during sleep, when there is normally a drop in sympathetic tone, it may be harmful. These dynamic skin perfusion responses during sleep cannot be detected by pulse oximetry. Figures 1-3 illustrate the relationship between the response of the molecular tissue oximeter and the response of a medical-grade pulse oximeter sensor relative to inhalation of nitrogen vs. inhalation of oxygen, and during and following physical exercise, as the basis of our belief that the molecular tissue oximeter is detecting skin tissue hypoxia and hyperoxia. 


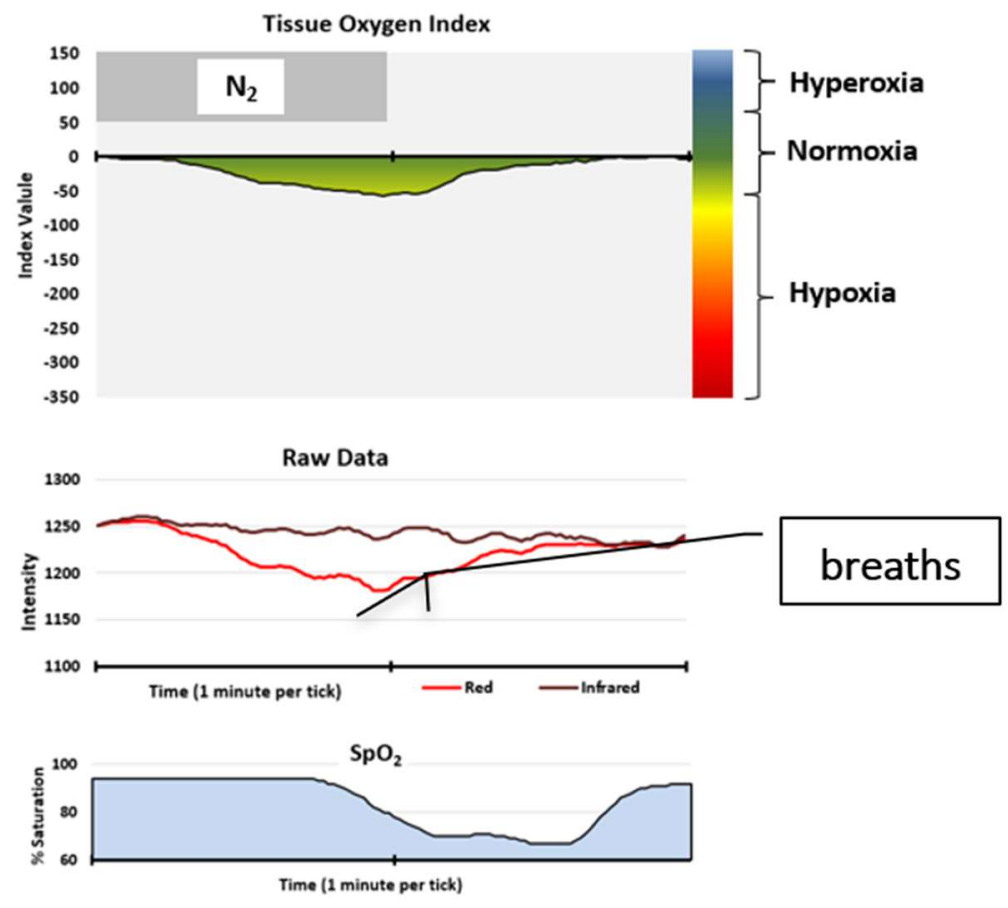

Figure 1. Tissue Oxygen Index (TOi) recording while breathing nitrogen $\left(\mathbf{N}_{2}\right)$ for 1 minute.

When less than the needed quantity of oxygen is being delivered to skin tissue, as induced experimentally by briefly breathing nitrogen gas, the molecular tissue oximeter sensor detects increased absorption of the red wavelength of sensor light, with little or no change in the absorption of the infrared light. The return to red light baseline detected intensity immediately follows relief of tissue hypoxia by again breathing air. The lack of data processing delay in the molecular tissue oximeter data delivery is likely to be found very important in optimizing the safety and effectiveness of oxygen therapy during surgical anesthesia and critical care. The variations in amplitude of the infrared signal are seen in real-time to correspond with breath cycles and offer an analog of the effort of each breath.

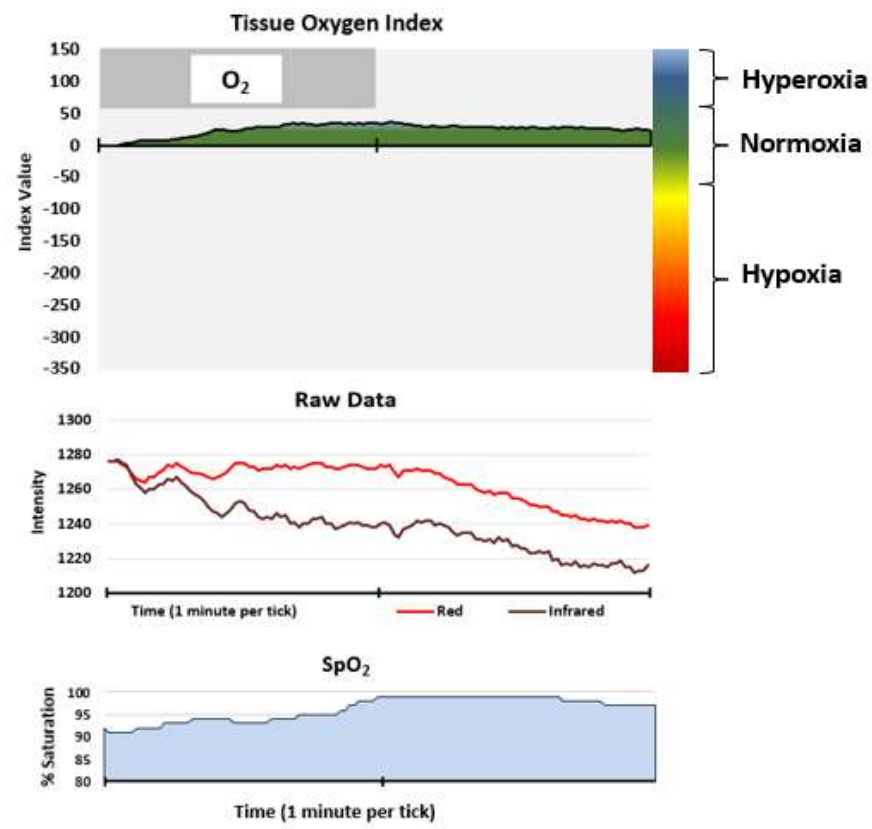

Figure 2. Tissue Oxygen Index recording while breathing $100 \%$ oxygen $\left(\mathrm{O}_{2}\right)$ for 1 minute. 
On the other hand, if excess oxygen is delivered to skin tissue, as induced by breathing $100 \%$ oxygen, the molecular tissue oximeter sensor detects increased absorption of the sensor's infrared wavelength, with a slight decrease in the absorption of the red light. When the sensor is in contact with the skin of the mid-upper arm, both of these photonic signal responses become evident within 5-10 seconds of the onset of changes in the partial pressure of $\underline{o x y g e n}_{\left(\mathrm{ppO}_{2}\right)}$ of the breathing gas. Due to extended averaging to suppress motion artifact, pulse oximeter data response may be delayed up to 45 seconds, as shown in Figures 1 and 2. Remarkably, prolonged exposure to excess oxygen in the breathing gas results in progressively increasing absorbance of the infrared light. This photonic response is prolonged for up to several hours after the excess oxygen exposure is discontinued and the subject returns to breathing air. Pulse oximeters cannot detect tissue hypoxia or hyperoxia.

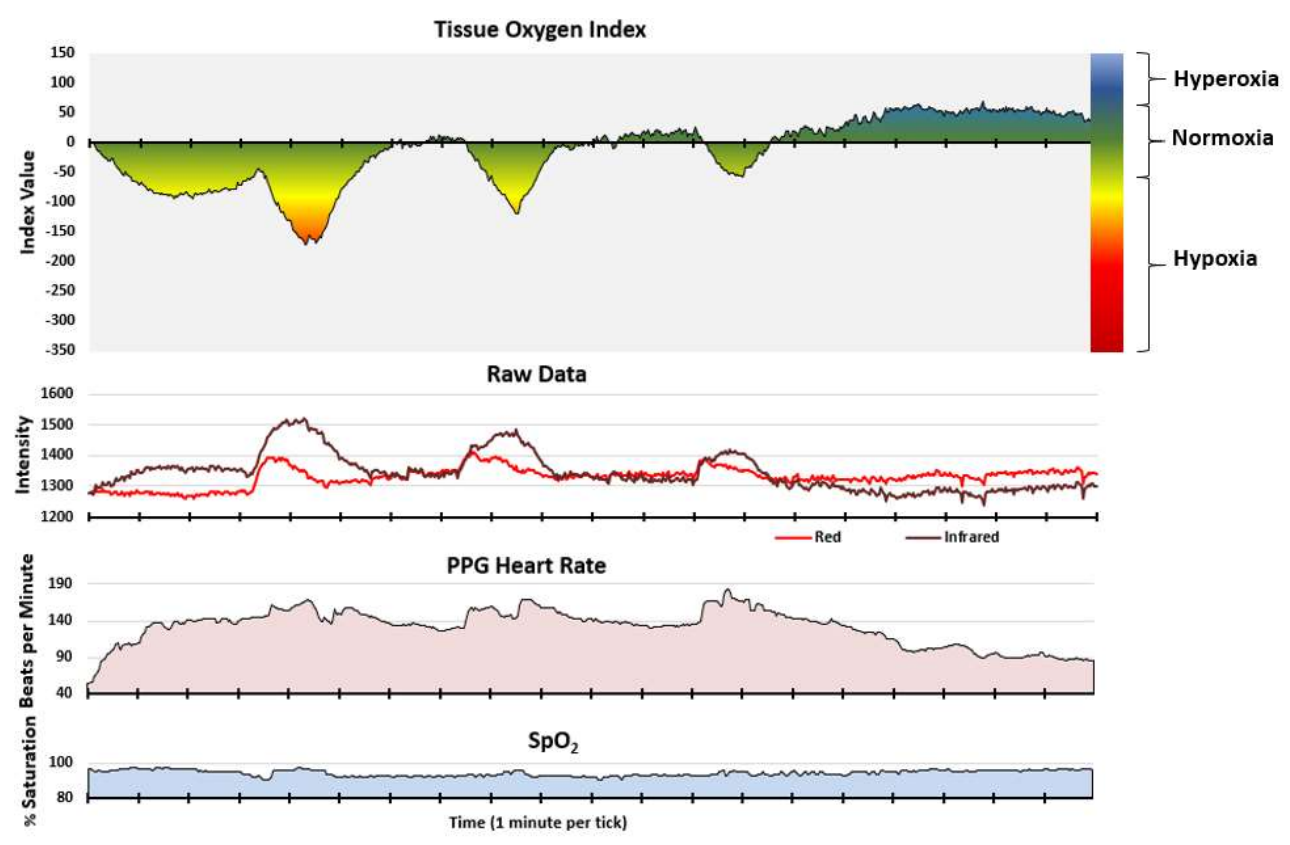

Figure 3. Simultaneous recording during exercise: Tissue Oxygen Index and the Raw Data from which it is derived, PPG Heart Rate, and $\mathrm{SpO}_{2}$.

With the molecular tissue oximeter, we have also gained new insights regarding physiologic responses in the skin during and following exercise. Figure 3 presents data recorded simultaneously during a stationary bicycle interval exercise session. The athlete's resting heart rate is in the upper 40 to low 50 beats per minute range. His maximum

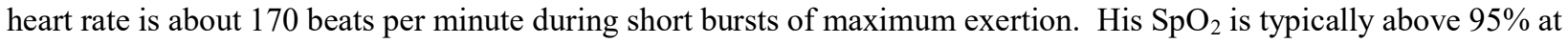
his living altitude of about 4200 feet AMSL and, as the $\mathrm{SpO}_{2}$ graph shows, does not vary significantly during exercise. During his warmup, however, the molecular tissue oximeter detects an immediate development of skin tissue hypoxia, likely due to vasoconstriction as a first-line defense against hypotension and hypoxemia as muscle blood flow and oxygen consumption increases. The molecular tissue oximeter detects this vasoconstriction in the skin by the reduced absorption from less blood in the sensor light path of both the infrared and red light; mostly infrared. During the successive three similar intervals of high exertion, the skin tissue hypoxia deepens significantly. However, there is a progressive lessening of the photonic responses with successive intervals of near-equal effort. Upon stopping exercise immediately following the third interval, the molecular tissue oximeter indicates that his skin tissue becomes hyperoxic, with relatively less absorption of the red wavelength, despite the fact that the blood volume in the sensor light path (infrared trace of the Raw Data) immediately returns to its baseline value range.

It is important to note that there are several commercial and medical device sensors that are called 'tissue oximeters' by their developers, which only measure the oxygen saturation of the blood flowing within the tissue. These devices typically use infrared light that can penetrate deeper than the skin to measure the average oxygen saturation of the blood hemoglobin flowing within the deeper tissues, such as muscle and brain. These are typically used to monitor increased 
consumption of oxygen by the tissue as indicated by lowering of the average blood oxygen saturation. Accurate measurement of the molecular oxygen tension, or oxygen content, within tissue, such as with a Clark electrode or by the Lumee ${ }^{\circledR}$ implant and sensor system (Profusa, Inc.) [15], respectively, has also been demonstrated. Movement of blood cells within capillaries in the skin is also detectable using Doppler-shifted laser light, as an index of blood perfusion in the skin, which is reduced under hypoxic stress by reflex vasoconstriction mechanisms. All of this information may be clinically important and useful as indices of cardiopulmonary health, of molecular oxygen supply to tissues, and of blood flow within tissue. However, the metabolic status of the tissue relative to the oxygen supply must be assumed. None of these sensors can indicate whether the tissue is receiving less than enough oxygen, just the right amount of oxygen, or too much oxygen.

The Reveal molecular tissue oximeter uses timed samples $(1-4 \mathrm{~Hz})$ of tissue-diffused and -absorbed light intensity at unique wavelengths and calculates its Tissue Oxygen Index (TOi) output data by subtracting the detected infrared intensity value from the detected red intensity value. This provides a continuous numeric scale, with a central, or zero, position at the awake, non-stressed condition, and extends with a negative trend with skin tissue hypoxia, and a positive trend with skin tissue hyperoxia. The wavelengths used were determined by spectroscopic analysis carried out during experiments mentioned above, including: (1) exposing individuals to conditions known to under- or over-supply oxygen to skin tissue, including breathing nitrogen or breathing 100\% oxygen, (2) during and following physical exertion, (3) during reperfusion of skin following a period of halted blood flow, and (4) during return to the previously normal blood oxygen saturation following an extended period of mild hypoxemia, such as during an airline flight. Results of these experiments with the molecular tissue oximeter, in parallel with pulse oximetry, clearly show skin tissue spectral absorption responses that are independent, to varying degrees, of simultaneously measured blood oxygen saturation. Recordings of multi-photon-stimulated fluorescence responses to induced skin tissue hypoxia indicate the dynamic status of energy conversion processes within tissue mitochondria [16]. While further study is needed to fully define the photonic sources of the molecular tissue oximeter's robust signal responses, the experimental evidence so far indicates that that oxygen supply-dependent energy conversion molecular phenomena are involved [17].

\section{TISSUE OXIMETER SLEEP TESTING}

The current wireless wearable Reveal molecular tissue oximeter sensor prototype has been used to record skin tissue oxygenation during sleep, with the sensor placed on the mid-upper arm. This device was initially used to record tissue oxygenation during sleep of an individual who had long been noted to be a loud snorer and to occasionally stop breathing during sleep. The recorded molecular tissue oximeter data indicated periodic skin tissue hypoxia throughout the night. Subsequent recording of molecular tissue oximetry data concurrently with PSG testing and, later, during therapy with auto-titrating continuous positive airway pressure (APAP) during sleep have confirmed the initial impression that this sensor was detecting SDB events.

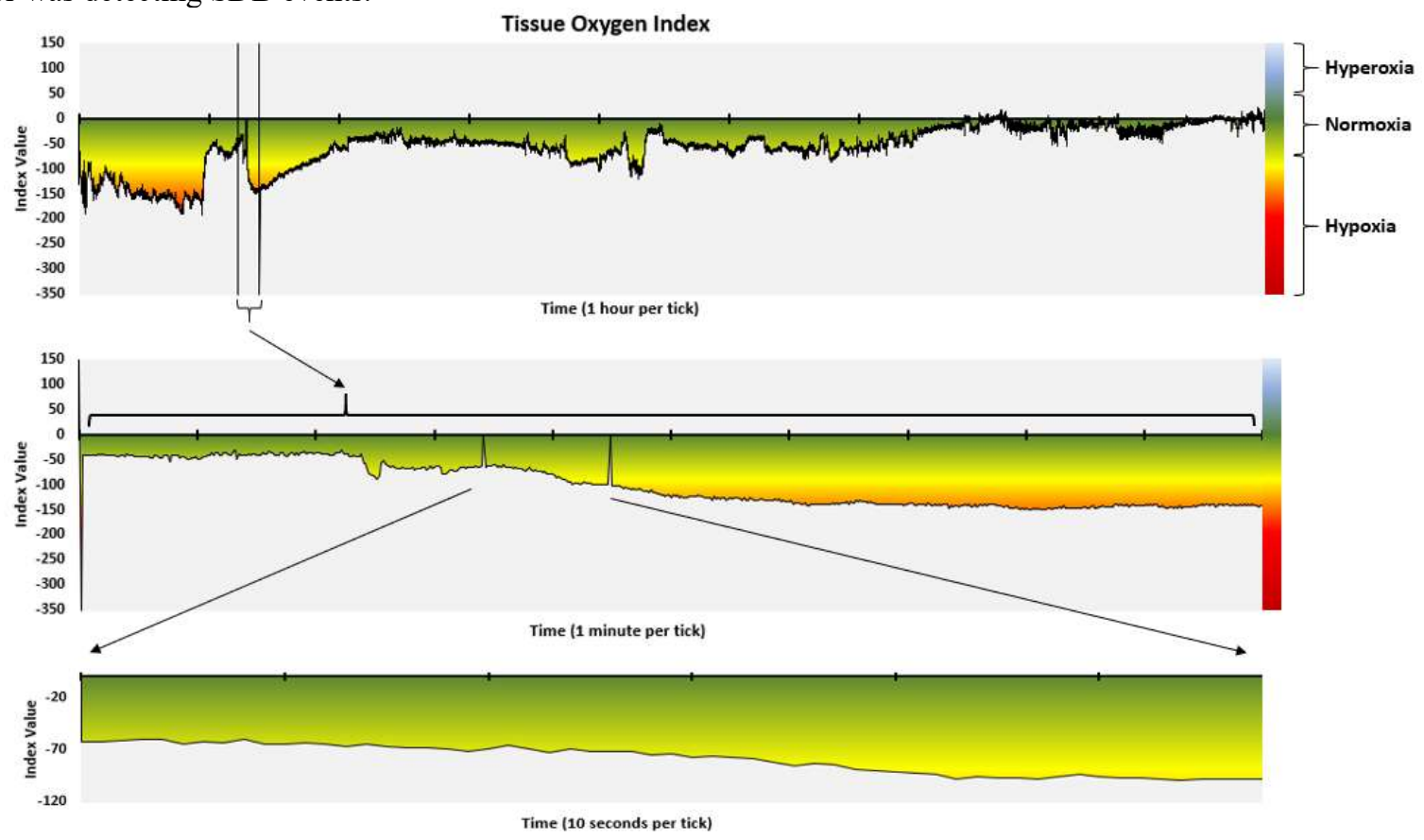

Figure 4. Tissue Oxygen Index sleep record. 
These three graphs in Figure 4 portray the Tissue Oxygen Index trend throughout a sleep study period. A segment is selected, based on the subject: being asleep; snoring, and without change in $\mathrm{SpO}_{2}$; but with a declining Tissue Oxygen Index trend. A sample sub-period within this segment is shown in the bottom graph and corresponds with the oneminute view of PSG data in Figure 5.

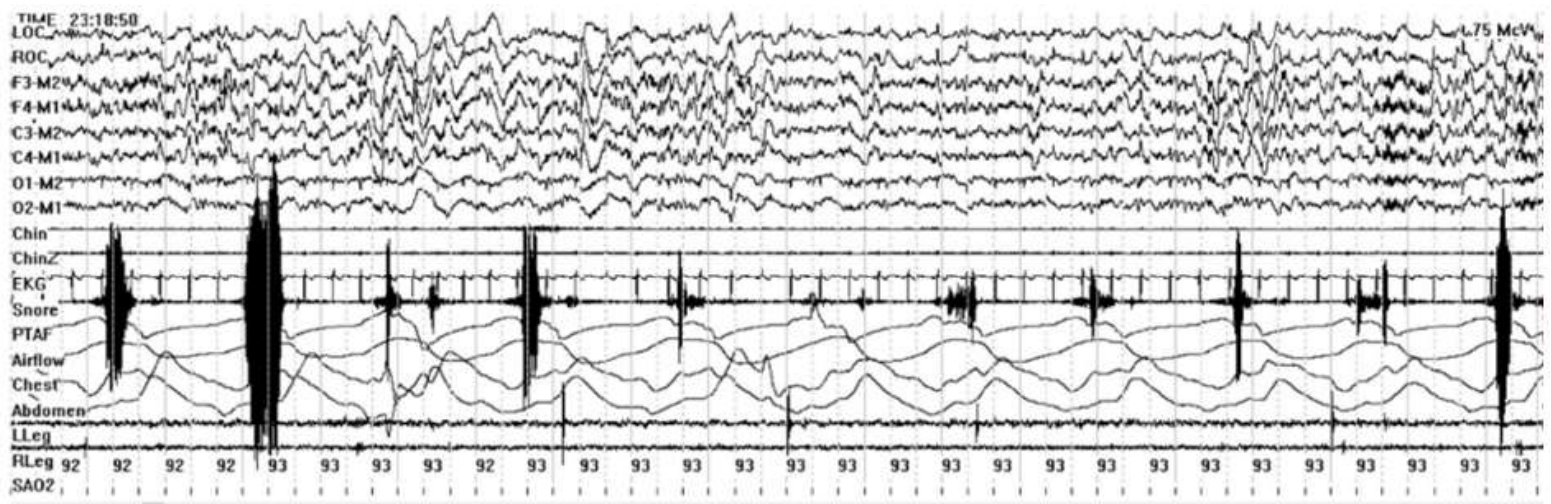

Tissue Oxygen Index

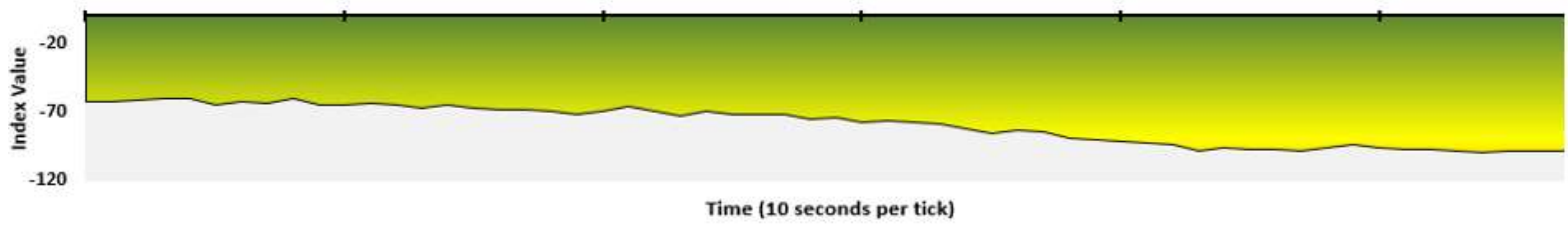

Figure 5. PSG and Tissue Oxygen Index during primary snoring.

The selected one-minute segment in Figure 5 shows decreasing TOi trend while the PSG sleep study indicates that the subject is: asleep; snoring; without pulse oximetry desaturation; without awakening; with only very minimal arousal; and without apnea, hypopnea or obvious hypoventilation. This selected segment is not the only such TOi deviation. This recording session also revealed extensive additional periods with skin tissue hypoxia which were not associated with decreased $\mathrm{SpO}_{2}$. Martinez-Garcia [13] presents current thinking about the effect of chronic sympathetic stimulation, when not relieved normally during sleep, that may be a root cause of refractory hypertension. As depicted in this recorded segment, skin tissue hypoxia during SDB, including primary snoring, as detected by the molecular tissue oximeter sensor, may be a useful indicator of abnormal sympathetic vasoconstriction in the skin during sleep. Further studies are needed to find if resolution of this phenomenon results in reduction, or better, prevention of refractory hypertension and its co-morbidities.

TOi recordings during sleep have also consistently detected cyclic variations in the infrared signal that correspond in time with each breath, and in amplitude with breathing effort. Real-time observation shows that this signal response is apparently due to breathing-induced variation in the venous blood volume in the skin within the sensor's light path. This response also appears to be an optically-detected analog of the blood flow basis of pulsus paradoxus, which is used clinically to detect increased breathing effort, such as during acute asthma attacks. The 'normal range' occurrence of pulsus paradoxus is systolic blood pressure variation of up to $10 \mathrm{mmHg}$ that occurs with each breathing cycle as the volume of venous blood in the large chest veins and right atrium cycles with each breath because of varying intrathoracic pressure. Restricted air flow, as with asthma, OSA, and chronic obstructive pulmonary disease (COPD), accentuate this venous blood shift and the resulting systolic blood pressure variation, making pulsus paradoxus a sensitive and useful index of the severity of the air flow restriction, and of the increased effort needed to breathe.

The molecular tissue oximeter sensor also detects the rostral shift of blood volume from the lower body as the blood redistributes throughout the length of the body over the first one to two hours after reclining. In addition, the sensor shows consistent patterns of blood volume shifts depending on the location of the sensor on the body relative to the heart. During sleep on one's side, when the sensor is located on the upper arm above the heart, there is relatively less venous blood in the skin at the sensor site, resulting in less absorption of the sensor's light. When the sensor is located lower than the heart, there is relatively more blood in the skin beneath the sensor, resulting in more light absorption. Regardless of body position, any insufficiency in the delivery of oxygen to the skin tissue at the sensor site is independently indicated by increased absorbance of the red light, with the corresponding infrared light signal remaining relatively unchanged. The TOi subtraction calculation removes the tandem variations of the red and infrared signals 
that occur with rostral blood volume shift, with breathing, and with sensor motion against the skin, to show just the relative redox status of the skin tissue. A negative TOi trend indicates progressive skin tissue hypoxia. The zero, +/50 , region is seen during the normally acclimated redox status of the skin tissue. A positive numeric trend beyond this indicates tissue hyperoxia. Breathing $100 \%$ oxygen with normal lungs typically raises the TOi value to over 200 . Skin tissue hypoxia, such as during prolonged, severe SDB events, is associated with TOi values as low as -350 . Signal display and data analysis includes the detected red and infrared intensity trends and the calculated TOi trend. OSA events cause sharp, tandem, positive-going 'spikes' in the red and infrared signals. Central sleep apnea (CSA) events result in diminished, or lack of, breathing-induced variation in the infrared signal relative to the normal breathing signal pattern while awake. Thus, breathing effort- and gravity-induced variations in blood volume in the skin beneath the sensor are readily monitored. However, of particular importance is the observation of skin tissue hypoxia being detected during primary snoring coincident with normal arterial blood oxygen saturation. This new sensor and data analysis method offers a potentially useful biometric index of hypoxic stress during primary snoring.

The possibility that skin tissue hypoxia, as detected by molecular tissue oximetry, could serve as a proximal measure of snoring-induced pathology, is consistent with known auto-regulatory mechanisms. The skin tissue hypoxia that is detected by the molecular tissue oximeter sensor in response to breathing air with decreased available oxygen, during exercise, and during apnea is consistent with well-known reflex responses that help maintain blood pressure and minimize decreases in oxygen saturation of the arterial blood supplying the brain and other vital organs at the expense of decreased oxygen supply to the skin. These reflex vascular responses include restricting blood flow to the skin; thus, maintaining blood pressure and prioritizing oxygen availability to vital organs and muscles [14]. The molecular tissue oximeter sensor, being able to non-invasively and continuously discern skin tissue oxygen need vs. supply, has been found to be a convenient, relevant and sensitive indicator of hypoxic challenges during sleep. Uniquely, it indicates skin tissue hypoxia in situations, such as during primary snoring, when the arterial blood oxygen saturation is maintained within normal limits by various reflex mechanisms, including the reduced skin perfusion that results in skin tissue hypoxia. We present evidence that molecular tissue oximetry of the skin is detecting physiologic stress occurring as a result of snoring that remains undetected by pulse oximetry. This represents a promising proximal measure of pathology occurring with primary snoring events. The same logic that suggests the potential utility of molecular tissue oximetry of the skin for detection of pathology associated with snoring supports that this methodology may also have utility as a more sensitive indicator of pathology associated with hypoventilation due to any cause (e.g. obesityhypoventilation syndrome) than currently available methods. The molecular tissue oximeter sensor is currently undergoing systematic validation to determine its clinical utility as a more sensitive means of detecting pathology associated with SDB than is currently available.

\section{SUMMARY}

We have reviewed the basis for molecular tissue oximetry and presented preliminary evidence that the Reveal molecular tissue oximeter sensor provides a convenient, non-invasive, and objective means of measuring skin tissue oxygenation that could potentially be useful in the Sleep Lab and for home testing for SDB. We have also found skin tissue hypoxia occurring during SDB, and during periods of primary snoring. This new marker of physiologic stress during primary snoring may be a key index of the pathophysiology leading to hypertension and, potentially, other comorbidities of primary snoring. Studies are needed to characterize the nature of the molecular tissue oximetry signal, to determine its clinical usefulness for assessment of SDB in general, and to evaluate its ability to more sensitively detect pathology occurring in association with snoring than available methods.

\section{REFERENCES}

[1] American Academy of Sleep Medicine. International Classification of Sleep Disorders. 3rd ed. Darien, IL: American Academy of Sleep Medicine; 2014

[2] Smith DL, Gozal D, Hunter SJ, Kheirandish-Gozal L. "Frequency of snoring, rather than apnea-hypopnea index, predicts both cognitive and behavioral problems in young children." Sleep Med. 2017 Jun; 34:170-178. PMID: 28522088

[3] Berry, R.B., Budhiraja, R., Gottlieb, D.J., Gozal, D., Iber, C., Kapur, V.K., Marcus, C.L., Mehra, R., Parthasarathy, S., Quan, S.F., Redline, S., Strohl, K.P., Davidson Ward, S.L., Tangredi, M.M., and American Academy of Sleep, M., "Rules for scoring respiratory events in sleep: update of the 2007 AASM Manual for the Scoring of Sleep and Associated Events"

[4] "Deliberations of the Sleep Apnea Definitions Task Force of the American Academy of Sleep Medicine." J Clin Sleep Med, 2012. 8(5): p. 597-619. PMCID: PMC3459210

[5] Iber, C., Ancoli-Israel, S., Chesson, A.L.J., and Quan, S.F., "The AASM manual for the scoring of sleep and associated events: rules, terminology and technical specifications." 2007, Westchester, IL: American Academy of Sleep Medicine.

[6] Lee, SK, et. al., "Increased risk of new-onset hypertension in midlife male snorers: The 14-year follow-up study." J Sleep Res 2018 Sep 3, PMID: 30252172

[7] Deeb, R, et. al., "Snoring and carotid artery disease: A new risk factor emerges." The Laryngoscope, 2018 Sep 8, PMID: 30194704 
[8] Dunietz, GL, et. al., “Associations of snoring frequency and intensity in pregnancy with time-to-delivery.” Paediatr Perinat Epidemiol, 2018 Sep 28, PMID: 30266041

[9] Wada, H, et. al. "Nocturnal enuresis and sleep disordered breathing in primary school children: Potential implications.” Pediatr Pulmonol, 2018 Sep 11, PMID: 30203928

[10] Scalzitti, NJ, et. al., "Diagnosis and perioperative management in pediatric sleep-disordered breathing." Paediatr Anaesth, 2018 Oct 3, PMID: 30281185

[11] Tamisier, R, et. al., "Sleep biology updates: hemodynamic and autonmic control in sleep disorders." Metabolism Clinical and Experimental 84 (2018) 3-10. PMID: 29572132

[12] Sasaki, N, et. al., "Associations between charactistics of obstructive sleep apnea and nocturnal blood pressure surge.” Hypertension, November 2018, 11331140, PMID: 30354806

[13] Martinez-Garcia, MA, et. al., "Beyond Resistant Hypertension,” Hypertension AHA, 2018 Sep;72(3):618-624, PMID: 30354751

[14] Kenney WL, et. al. "Blood pressure regulation III: what happens when one system must serve two masters: temperature and pressure regulation?" Eur J Appl Physiol. 2014 March ; 114(3): 467-479. doi:10.1007/s00421-013-2652-5, PMID: 23636697

[15] Lumee (Profusa, Inc.) Skin tissue oxygen level varies relative to exercise/rest: [https://profusa.com/news-and-events/health-risk-management-value-real-timemonitoring-body-chemistries/]

[16] Balu M, et. al., "In vivo multiphoton NADH fluorescence reveals depth-dependent keratinocyte metabolism in human skin.” Biophysical Journal 2013 Jan 8;104(1):258-67, PMID: 23332078

[17] Hatch, GM, et. al., “Molecular Tissue Oximeter,” Reveal Biosensors, 2018 [https://drive.google.com/open?id=1OyqjpOGassmT1PSN6Mx-AEDdz2j4RrTx] 